\section{Reduced lipolysis as possible cause for greater weight gain in subjects with the Pro12Ala polymorphism in PPAR $\gamma_{2}$ ?}

To the editor: We read with interest the letter by Lindi et al. in the July issue of Diabetologia, reporting the influence of the common Pro12Ala polymorphism in the peroxisome-proliferator-activated receptor - PPAR $\gamma_{2}$ - on long-term weight change [1]. Over a 10-year period those who were carriers of the Ala12 allele in PPAR $\gamma_{2}$ gained substantially more weight than those with 2 Pro alleles. Association studies of genetic polymorphisms using longitudinal data sets contain far more information than cross-sectional studies. Therefore, the data by Lindi et al. adds substantially to the equivocal evidence obtained by cross-sectional analysis regarding the influence of this polymorphism on obesity.

The authors point out that the polymorphism is also associated with greater insulin sensitivity and propose that this could be linked with the greater increase in body weight. We would like to contribute to this discussion by referring to recently published data of ours showing that the PPAR $\gamma_{2}$ Prol2Ala polymorphism is associated with greater insulin sensitivity of lipolysis [2]. In addition, we present data based on three times as many patients.

In our study, we used an isotopic approach (deuterium-labelled glycerol) in 68 normal glucose tolerant (NGT) subjects to determine the rate of appearance of glycerol as an index for systemic lipolysis. This has an advantage over measuring the turnover of non-esterified fatty acids (NEFA) which, in contrast to glycerol, can be re-esterified to triglycerides in adipose tissue. During a three-step hyperinsulinaemic clamp, we observed a significantly smaller insulin $\mathrm{EC}_{50}$ of lipolysis suppression in carriers of the Ala12 allele suggesting greater insulin sensitivity of lipolysis [2]. Because the technique used only measures the systemic rate of appearance of glycerol we could not make any inference regarding specific regional fat depots.

For this purpose, we re-analysed standard hyperinsulinaemic-euglycaemic clamp data from the database of the Tübingen family (TüF) study of patients with Type II (non-insulin-dependent) diabetes mellitus. We specifically selected unrelated NGT subjects in whom NEFA concentrations were measured during the clamp $(n=177)$. Details of the methods have been described previously $[2,3]$. We used the glucose infusion rate required to maintain euglycaemia (expressed per kilogram lean body mass) as a measure of insulin sensitivity. Table 1 shows the anthropometric and metabolic characteristics of the two genotype groups (39 subjects heterozygous and 3 subjects ho- mozygous for the polymorphism were pooled and referred to as $X / A l a)$. Data are means \pm SEMs and statistical comparisons were carried out by the non-parametric Wilcoxon rank test.

The baseline parameters in the two groups were virtually identical. However, in contrast to fasting NEFAs, the suppressed NEFA concentrations during the clamp were almost $50 \%$ lower $(p=0.02)$ in patients with the polymorphism. This indicates greater insulin sensitivity of lipolysis and confirms our previous report [2]. The more efficient suppression of lipolysis by insulin in the Pro12Ala group could be part of the mechanisms leading to the significantly greater weight gain. More efficient suppression of lipolysis could tilt the minutely regulated balance between lipolysis and lipogenesis slightly towards lipogenesis. This would result in overproportionate retention of NEFAs in stored triglycerides during insulin stimulation, as opposed to release during insulin deprivation (fasting), and in the long-run favour weight gain.

How can favouring of obesity on the one hand be reconciled with reduced risk for Type II diabetes [4] on the other hand? A possible explanation might involve compositional (i.e. size and number of fat cells) and metabolic differences of the adipose tissue between the genotypes. Adipose tissue triglycerides primarily represent a storage form of energy from whatever source (glucose, lipids, even some amino acids). Conceivably, the excess adipose tissue might provide a "metabolically forgiving" sink for excess energy (due to overeating) in subjects with the polymorphism. Thus, less harmful, albeit larger, fat depots might accumulate but prevent perturbation of glucose homoeostasis for quite some time. Reports on lipatrophic diabetes in which weight gain with thiazolidinedione treatment is accompanied by metabolic improvement support this [5]. Moreover, heterozygous $P P A R \gamma$-deficient mice were protected from the development of insulin resistance due to adipocyte hypertrophy under a high-fat diet [6]. At this point, however, the cellular mechanism which links altered transcriptional activity of $P P A R \gamma_{2}$ Ala12 [7] with metabolic effects in humans in vivo is not clear. Effects of fat distribution (subcutaneous vs intra-abdominal [8]) or fat cell size (smaller vs larger [6]) could be involved.

In summary our finding of reduced NEFA release in subjects with $P P A R \gamma_{2}$ Pro12Ala polymorphism could be a partial explanation for their greater weight gain.

\section{Stumvoll, H. Häring}

\section{References}

1. Lindi V, Sivenius K, Niskanen L, Laakso M, Uusitupa MI (2001) Effect of the Pro12Ala polymorphism of the PPAR-

Table 1. Characteristics of subjects without (Pro/Pro) and with $(X / A l a)$ the Pro12Ala polymorphism in $P P A R \gamma_{2}$

\begin{tabular}{|c|c|c|c|}
\hline & Pro/Pro & $\mathrm{X} / \mathrm{Ala}$ & $p$-value ${ }^{\mathrm{a}}$ \\
\hline$n$ (male/female) & $135(61 / 74)$ & $42(21 / 21)$ & $0.5^{\mathrm{b}}$ \\
\hline Age (years) & $32 \pm 1$ & $33 \pm 2$ & 0.5 \\
\hline Weight & $74.2 \pm 1.4$ & $74.2 \pm 1.9$ & 1.0 \\
\hline Waist-to-hip ratio & $0.83 \pm 0.01$ & $0.83 \pm 0.01$ & 0.8 \\
\hline Fasting plasma glucose $(\mathrm{mmol} / \mathrm{l})$ & $4.87 \pm 0.04$ & $4.99 \pm 0.06$ & 0.10 \\
\hline Fasting plasma insulin $(\mathrm{pmol} / \mathrm{l})$ & $46 \pm 2$ & $42 \pm 5$ & 0.5 \\
\hline Glucose infusion rate $($ clamp $)(\mathrm{mg} /[\mathrm{kg}$ lean body mass $\cdot \min ])$ & $8.7 \pm 0.3$ & $9.1 \pm 0.4$ & 0.4 \\
\hline
\end{tabular}

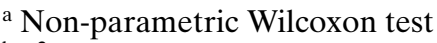

b $\chi^{2}$-test

${ }^{c}$ Measured at the end of the clamp (120 min) 
$\mathrm{g}_{2}$ gene on long-term weight change in Finnish non-diabetic subjects. Diabetologia 44: 925-926

2. Stumvoll M, Wahl HG, Löblein K et al. (2001) The Pro12Ala polymorphism in the peroxisome proliferator activated receptor $\mathrm{g} 2$ gene is associated with increased antilipolytic insulin sensitivity. Diabetes 50: 876-881

3. Stumvoll M, Wahl HG, Löblein K et al. (2001) A novel use of the hyperinsulinemic-euglycemic clamp technique to measure insulin sensivity of systemic lipolysis. Horm Metab Res 33: 89-95

4. Altshuler D, Hirschhorn JN, Klannemark Met al. (2000) The common PPAR gamma Pro12Ala polymorphism is associated with decreased risk of type 2 diabetes. Nat genet 26: 76-80

5. Reitman ML, Arioglu E, Gavrilova O, Taylor SI (2000) Lipoatrophy revisited. Trends Endocrinol Metab 11: 410-416

\section{A novel hepatocyte nuclear factor-1及 (MODY-5) gene mutation in an Italian family with renal dysfunctions and early-onset diabetes}

To the Editor: Mutations in the homeodomain-containing transcription factor hepatocyte nuclear factor $(H N F)-1 \beta$ have been reported in association with maturity-onset diabetes of the young (MODY-5) and early-onset and progressive non-diabetic renal dysfunction [1-2]. Iwasaki et al. recently screened the $H N F-1 \beta$ gene for mutations in a Japanese family whose members had $H N F-1 \beta$ diabetes, renal dysfunction and abnormalities of genital development [3]. They found a new heterozygous mutation in the hepatocyte nuclear factor $(H N F)-1 \beta$ located in the splice donor site of exon 2 , associated with these different clinical phenotypes. In addition, the results of Weng et al. suggested that $H N F-1 \beta$ mutations might play an important role in the development of renal disease [4].

Here, we report a three-generation Italian family in which a syndrome of diabetes and severe non-diabetic renal disease is associated with a novel deletion mutation in the $H N F-1 \beta$ gene (Fig. 1).

The index subject (III-1) was a girl with polycystic kidneys who was followed from the birth. During follow-up the patient showed incidental hyperglycaemia and impaired glucose tolerance on OGTT, at 12 years of age. After kidney transplantation, at 13 years of age she developed diabetes and insulin therapy started.

The index subject's two younger sisters (III-2 and III-3) had small kidneys with occasional cysts and a normal glucose tolerance test.

Her father (II-2) had horseshoe kidney and after renal surgical removal chronic renal insufficiency (CRI) developed. When the father was 20 years old, he was diagnosed as having diabetes and was treated with insulin.

All these subjects underwent immunologic (islet cell autoantibodies, such as IAA, GADA and IA-2A) and immunogenetic (HLA typing) markers for Type I (insulin-dependent) diabetes mellitus. No autoantibodies were present in the subjects; regarding DQA1 and DQB1 typing, one heterodimer was present in the two sisters and in the father, no heterodimers were in the index subject.

Moreover the paternal aunt (II-1) and grandmother (I-2) of the index subject showed renal dysplasia with renal insufficiency without history of diabetes.

Therefore, renal manifestations are variable but are a constant feature in all six members of this family; until now only
6. Kubota N, Terauchi Y, Miki H et al. (1999) PPAR gamma mediates high-fat diet-induced adipocyte hypertrophy and insulin resistance. Mol Cell 4: 597-609

7. Deeb SS, Fajas L, Nemoto M et al. (1998) A Pro12Ala substitution in PPARgamma2 associated with decreased receptor activity, lower body mass index and improved insulin sensitivity. Nat Genet 20: 284-287

8. Adams M, Montague CT, Prins JB et al. (1997) Activators of peroxisome proliferator-activated receptor gamma have depot-specific effects on human preadipocyte differentiation. J Clin Invest 100: 3149-3153

Corresponding author: M. Stumvoll, Medizinische Universitätsklinik, Otfried-Müller-Str. 10, Tübingen, Germany, e-mail: michael.stumvoll@med.uni-tuebingen.de

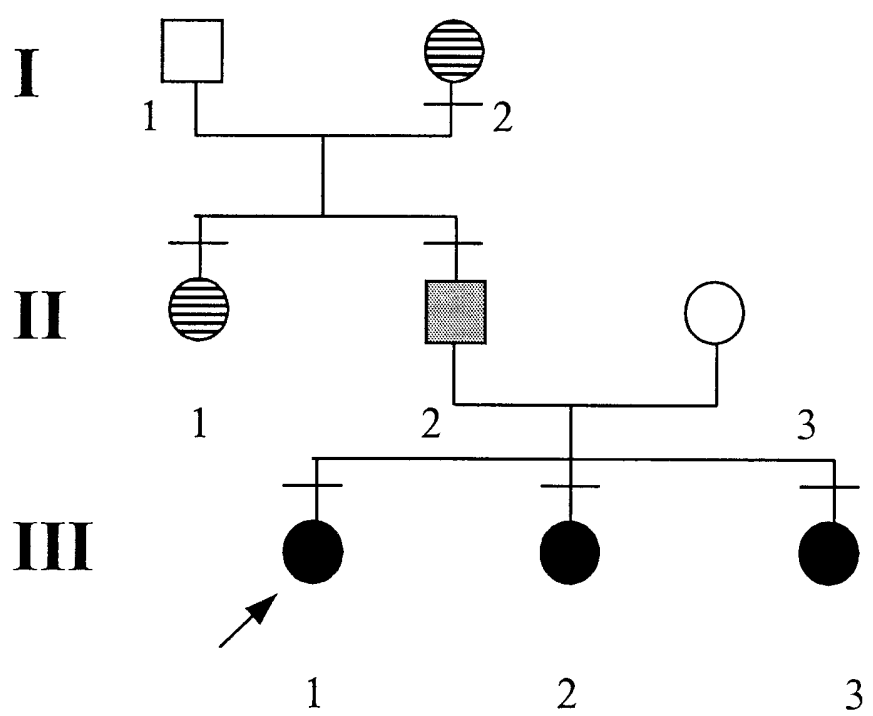

Fig. 1. A family tree showing clinical details and inheritance of $H N F-1 \beta$ mutation. The index subject (III-1), with bilateral renal cysts and diabetes diagnosed at 13 years of age, is indicated by an arrow. The proband's father (II-2) with horseshoe kidney and diabetes diagnosed at 20 years is represented by a grey square. Heterozygous mutation HNF-1ß (black line); Horseshoe kidney (solid square); Bilateral renal cysts (solid circle); Displastic kidneys (striped circle)

two had early onset diabetes, diagnosed before 25 years of age, without immunologic and immunogenetic markers for Type I diabetes susceptibility.

DNA was isolated from peripheral blood from these six patients in order to evaluate the $H N F-1 \beta$ gene.

The minimal promoter and coding regions of the nine exons and flanking introns of the $H N F-1 \beta$ gene were screened for mutations by direct sequencing of PCR products using specific primers as described [1]. The novel mutation was confirmed by cloning the PCR product into the vector pCR2.1TOPO (Invitrogen, Carlsbad, Calif., USA) and sequencing clones derived from both alleles.

We found an heterozygous deletion in the splice donor site of intron 2 of the $H N F-1 \beta$ gene, located after the conserved GT-dinucleotide (designated IVS2 + 2delaagt), which might result in abnormal splicing of HNF- $1 \beta$ mRNA. This issue is currently under investigation and functional studies on the DNA transcripts and the encoded HNF- $1 \beta$ protein are in progress. 